\title{
Comportamiento reproductivo, forrajeo y uso de hábitat de Coccyzus ferrugineus (Cuculiformes: Cuculidae) en la Isla del Coco, Costa Rica
}

\author{
Javier Tenorio $^{1,2 *}$, Carmen Hidalgo ${ }^{1}$, Michel Montoya ${ }^{3}$ \& Oscar Ramírez-Alán ${ }^{1}$ \\ 1. Escuela de Ciencias Biológicas, Universidad Nacional, Heredia, Costa Rica; tenoriosp192@gmail.com, \\ chidalgo@una.cr, osoramirez@gmail.com \\ 2. Rò Brù Conservation Fund, Costa Rica. \\ 3. Asociación Ornitológica de Costa Rica, Amigos de la Isla del Coco; michelmontoyam@gmail.com \\ * Correspondence
}

Recibido 30-I-2019. Corregido 10-V-2019. Aceptado 16-IX-2019.

\begin{abstract}
Reproductive behavior, foraging and habitat use of Coccyzus ferrugineus (Cuculiformes: Cuculidae), Isla del Coco, Costa Rica. Introducción: Coccyzus ferrugineus is the least studied endemic bird of Isla del Coco, many aspects of its natural history and behavior remain unknown, being one the least known species in Costa Rica. Objective: To describe the reproductive behavior, foraging and habitat use of C. ferrugineus to generate information for making conservation decisions for the species. Methods: Direct behavioral observations (foraging, courtship, vocalization, feeding and intercourse) were made in plots between 0-400 m above sea level, during two observation periods (morning and afternoon). A description of the foraging behavior and the reproductive cycle is presented. Results: $C$. ferrugineus uses the entire altitudinal gradient of the island to reproduce, from sea level to $400 \mathrm{~m}$ altitude in the cloud forest. Its reproductive season is marked by the beginning of the dry season. No significant differences were found between the established behavior categories and the time of day (Fisher, $\mathrm{P}>0.05$ ). However, significant differences were shown for the behavioral categories and the vertical stratum (Fisher, $\mathrm{P}<0.05$ ) demonstrating that it uses the entire vertical stratum of the vegetation structure. Conclusions: This study represents the basis for the knowledge of the reproductive biology and natural history of $C$. ferrugineus, it is urgent to carry out more studies on this species to be able to protect it and conserve the terrestrial ecosystem of Isla del Coco.
\end{abstract}

Key words: Breeding behavior; habitat use; Isla del Coco; Coccyzus ferrugineus; endemism; Costa Rica; conservation.

Tenorio, J., Hidalgo, C., Montoya, M., \& Ramírez-Alán, O. (2020). Comportamiento reproductivo, forrajeo y uso de hábitat de Coccyzus ferrugineus (Cuculiformes: Cuculidae) en la Isla del Coco, Costa Rica. Revista de Biología Tropical, 68(Supl. 1), S163-S176.

La Isla del Coco es uno de los sitios con mayor endemismo en Costa Rica, se conoce que alrededor del $11 \%$ del total de las especies de la isla son endémicas (Trusty, Kesler, \& Delgado, 2006; Zamora, 2008). La mayoría de las especies de aves nativas se encuentran amenazadas por especies alóctonas tanto de flora (103 especies) (Montoya, 2016), como de fauna, tales como la rata negra (Rattus rattus), la rata parda ( $R$. novergicus), el cerdo cimarrón (Sus scrofa), el gato doméstico (Felis catus) y el venado cola blanca (Odoicoleus virginianus), las cuales actúan como depredadoras y vectores de patógenos, provocando interferencia en la competencia interespecífica y desplazando a las especies nativas (Montoya, 2004; Tebbich, Taborsky, Fessl, Dvorak, \& Winkler, 2004; Orias, 2012; Medina, Bonnaud, 
Vidal, \& Nogales, 2014; Fourriére, AzofeifaSolano, \& Sandoval, 2016). A pesar de que esta amenaza se considera uno de los principales problemas que afectan al ecosistema terrestre de la isla (Dawson et al., 2015; Acosta-Vargas, 2016) el impacto de las especies introducidas ha sido poco analizado para las aves endémicas de la Isla del Coco (Fourriére et al., 2016).

Las principales especies que anidan en la Isla del Coco son Anous stolidus, A. minutus, Gygis alba, Onychoprion fuscatus, Fregata minor, Sula dactylatra, S. leucogaster, S. sula, Nesotriccus ridgwayi, Setophaga petechia aureola, Pinaroloxias inornata y Coccyzus ferrugineus. La Isla del Coco es además un sitio de descanso para otras 137 especies de aves, todas con algún grado de vulnerabilidad a factores antropogénicos y muy poco estudiadas (Fourriére et al., 2016). Esta falta de conocimiento impide que se efectúe un correcto plan de manejo para la conservación de estas especies y del ecosistema terrestre de la Isla del Coco.

Los miembros del género Coccyzus, familia Cuculidae, muestran similitudes en comportamiento, desarrollo y morfología incluyendo comportamientos de forrajeo, preferencia por las orugas, sistemas de construcción de nido, alimentación de cortejo, entre otras (Slud, 1964; Ralph, 1975; Stiles \& Skutch, 1989; Hughes, 2006; Martínez et al., 2011). El Cuclillo de la Isla del Coco (C. ferrugineus) es el ave endémica menos estudiada de la Isla del Coco y una de las especies menos conocidas (Stiles \& Skutch, 1989). Aún se desconocen muchos aspectos sobre su historia natural, por lo que este estudio representa la base fundamental para futuras investigaciones y aporta el conocimiento necesario preliminar para promover su conservación.

Por lo anterior, es necesario contar con información sobre la descripción del comportamiento de forrajeo, uso de hábitat y ciclo reproductivo que evidencie el uso y capacidad de la especie para la obtención de recursos que la abastecen de energía, sitios de descanso y de nidificación (Cueto, 1996; Avalos, 2009). El objetivo de este estudio fue describir el comportamiento reproductivo, de forrajeo, y el uso de hábitat de C. ferrugineus para generar información para la toma de decisiones de conservación de la especie.

\section{MATERIALES Y MÉTODOS}

Área de estudio: El estudio se desarrolló en la Isla del Coco, coordenadas $05^{\circ} 33^{\prime} 26^{\prime \prime}$ -

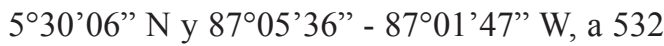
km de Cabo Blanco en Costa Rica continental, del 12 de febrero al 11 de marzo de 2017. Para un total de 203 horas de muestreo divididas en 29 días efectivos. El área terrestre comprende $24 \mathrm{~km}^{2}$ y el área marina $972.35 \mathrm{~km}^{2}$ con variaciones altitudinales que van desde los 0 m.s.n.m. hasta los 575 m.s.n.m. (AcostaVargas, 2016; Montoya, 2016). Es una isla oceánica formada por procesos volcánicos en la cordillera submarina, situada sobre la placa de Cocos con una edad estimada entre 1.91 y 2.44 millones de años (Bellon, Saenz, \& Tournon, 1983).

El ecosistema terrestre de la Isla del Coco es mayoritariamente Bosque Tropical lluvioso y Bosque Tropical Nuboso, siendo la única isla oceánica del Pacífico Tropical del Este que presenta condiciones de alta precipitación entre 5000-7 $000 \mathrm{~mm}$ anuales. El promedio anual de temperaturas a nivel del mar es de $25.5^{\circ} \mathrm{C}$, con un gradiente térmico de $0.4{ }^{\circ} \mathrm{C}$ con promedio anual de temperaturas mínimas de $23{ }^{\circ} \mathrm{C}$ y máximas de $27.6{ }^{\circ} \mathrm{C}$ (Montoya, 2016).

Descripción del hábitat: Se establecieron dos parcelas de 20 × 20 m cada 200 metros altitudinales, desde los 0 hasta los 400 m.s.n.m., para un total de 6 parcelas o $2400 \mathrm{~m}^{2}$. En cada parcela se midió la altura y el diámetro a la altura del pecho (DAP), con un clinómetro y cinta diamétrica respectivamente, a todos los árboles y arbustos que presentaran un DAP mayor o igual a $5 \mathrm{~cm}$, esto para categorizar la vegetación en tres estratos verticales (bajo, medio y alto). Además, se determinó el porcentaje de densidad foliar con un densiómetro, la riqueza relativa de árboles y arbustos y 
la temperatura con un termómetro ambiental de mercurio.

Descripción del uso del hábitat: La descripción del uso de hábitat se realizó en función de la estructura de la vegetación. Se determinó la presencia-ausencia de C. ferrugineus mediante observaciones directas en las parcelas clasificando el estrato en donde eran observados los individuos. Al mismo tiempo, se hicieron observaciones del comportamiento de la especie utilizando la metodología ad libitum e individuo focal (Altmann, 1974). Las observaciones de comportamiento se realizaron dividiendo los periodos de observación en dos intervalos: de las 0800 a las 1200 horas y de 1300 a las 1600 horas manteniendo una distancia mínima de $25 \mathrm{~m}$ con respecto a los individuos de $C$. ferrugineus observados para no alterar su comportamiento y evitar estresar a los animales.

El comportamiento se clasificó en cinco categorías: forrajeo, cortejo, vocalización, alimentación y cópula, definidos de la siguiente manera:

Forrajeo: Búsqueda intensiva de alimento, el individuo escarba con patas y pico en árboles, arbustos, palmeras, vegetación epífita, suelo y mediante vuelos rápidos.

Cortejo: Conjunto de movimientos corporales, despliegues y vocalizaciones del macho hacia la hembra para atraerla sexualmente. Este puede ir acompañado de danzas, forrajeo y regalos nupciales.

Cópula: Oposición de cloacas para la transferencia de esperma.

Vocalización: Emisión de vocalizaciones (cantos o graznidos) por uno o más individuos con el objetivo de comunicarse acústicamente.

Alimentación: Ingesta efectiva de alimento posterior a un forrajeo.

Descripción del comportamiento de forrajeo: Se realizaron observaciones directas utilizando el método ad libitum y el de individuo focal (Altmann, 1974) tomando en cuenta el número de individuos que forrajeaban simultáneamente y el estadio de cada individuo (juvenil-adulto) manteniendo una distancia mínima de $25 \mathrm{~m}$ con respecto a los individuos observados para no alterar su comportamiento y evitar estresar los individuos (Lehner, 1996). Cada vez que el individuo focal se desplazó más de $100 \mathrm{~m}$ o pasó inactivo de una observación más de 60 segundos se tomó como una nueva observación (Lehner, 1996; Martin \& Bateson, 2007).

Se aplicó una prueba de independencia por medio de la prueba de Fisher para determinar si la hora del día influye en el patrón de comportamiento de C. ferrugineus y en el uso del estrato vertical. Esta misma prueba se realizó para determinar si el patrón de comportamiento se relacionó con algún estrato de vegetación específico. Todos los análisis estadísticos se corrieron utilizando el software estadístico $\mathrm{R}$ versión 3.4.0.

Descripción del ciclo reproductivo: Para no afectar el éxito reproductivo ni el comportamiento de los individuos, el registro de los comportamientos se llevó a cabo mediante la búsqueda intensiva y el método de observación directa a una distancia mínima de $25 \mathrm{~m}$, determinando por medio de frecuencias los tiempos de cada comportamiento previamente establecidos, así como cualquier otro comportamiento inusual observado mediante la metodología ad libitum y de individuo o grupo focal (Altmann, 1974; Lehner, 1996; Martin \& Bateson, 2007). Con esto se evitó la atención de depredadores y el gasto innecesario de cuidado parental para protección del nido. Adicionalmente se realizó el presupuesto de tiempo para la especie durante la época reproductiva y se tomó la ubicación geográfica en donde se registraron a los individuos de C. ferrugineus.

\section{RESULTADOS}

Descripción del hábitat: La estructura de la vegetación difirió marcadamente entre los gradientes altitudinales (Tabla 1). A los 400 m.s.n.m. se encontró un bosque maduro, siendo esta zona altitudinal la que presentó los árboles 
TABLA 1

Temperatura y estructura de la vegetación por gradiente altitudinal de las parcelas colocadas para el estudio de Coccyzus ferrugineus (Cuculiformes: Cuculidae), Parque Nacional Isla del Coco. Se muestran promedios \pm desviación estándar

TABLE 1

Temperature and vegetation structure by altitudinal gradient of the sampled plots to study Coccyzus ferrugineus (Cuculiformes: Cuculidae), Isla del Coco National Park.Mean \pm standard deviation is shown

\begin{tabular}{|c|c|c|c|c|c|c|c|c|}
\hline $\begin{array}{c}\text { Altitud } \\
\text { (m.s.n.m.) }\end{array}$ & $\begin{array}{l}\text { Ubicación geográfica } \\
\text { de cada parcela }\end{array}$ & $\begin{array}{l}\text { Temperatura } \\
\text { mañana }\left(\mathrm{C}^{\circ}\right)\end{array}$ & $\begin{array}{c}\text { Temperatura } \\
\text { tarde }\left({ }^{\circ} \mathrm{C}\right)\end{array}$ & $\begin{array}{l}\text { Densidad } \\
\text { foliar }(\%)\end{array}$ & $\mathrm{DAP}(\mathrm{cm})$ & $\begin{array}{c}\text { Altura } \\
\text { vegetación } \\
\text { (m) }\end{array}$ & $\begin{array}{c}\text { Altura } \\
\text { mínima } \\
\text { vegetación } \\
(\mathrm{m})\end{array}$ & $\begin{array}{c}\text { Altura } \\
\text { máxima } \\
\text { vegetación } \\
\text { (m) }\end{array}$ \\
\hline 0 & $\begin{array}{l}05^{\circ} 32^{\prime} 38,6^{\prime \prime} \mathrm{N} 87^{\circ} 03 ’ 23,6^{\prime \prime} \mathrm{W} \\
05^{\circ} 32^{\prime} 39.0^{\prime \prime} \mathrm{N} 87^{\circ} 03^{\prime} 16.7^{\prime \prime} \mathrm{W}\end{array}$ & $28.0 \pm 1.41$ & $31.5 \pm 3.53$ & 92.6 & $15.42 \pm 16.55$ & $8.51 \pm 3.18$ & 2.6 & 20 \\
\hline 200 & 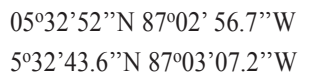 & $26.5 \pm 2.12$ & $26.5 \pm 0.70$ & 78.0 & $32.73 \pm 29.8$ & $11.86 \pm 4.89$ & 5 & 21 \\
\hline 400 & $\begin{array}{l}05^{\circ} 31^{\prime} 49.11^{\prime \prime} \mathrm{N} 87^{\circ} 03 \text { '59.7”W } \\
05^{\circ} 31^{\prime} 50.2^{\prime \prime} \mathrm{N} 87^{\circ} 04^{\prime} 010^{\prime \prime} \mathrm{W}\end{array}$ & $26.7 \pm 0.35$ & $26.5 \pm 0.70$ & 83.2 & $41.34 \pm 35.15$ & $15.88 \pm 7.92$ & 5 & 27 \\
\hline
\end{tabular}

más altos y con mayor DAP (Tabla 1). El piso altitudinal intermedio correspondiente a los $200 \mathrm{~m}$ de altitud representa una zona de transición con parches de regeneración entre la zona costera fragmentada y el bosque nuboso maduro. Los estratos verticales correspondieron a las alturas mínimas y máximas encontradas en cada piso altitudinal (Tabla 1). Así, el estrato vertical bajo correspondió a los árboles y arbustos entre 1 a $9 \mathrm{~m}$, el estrato vertical medio de los 10 a los $19 \mathrm{~m}$ y el estrato vertical alto abarcó todos aquellos árboles que presentaron alturas mayores a los $20 \mathrm{~m}$.

En la zona costera, a los 0 m.s.n.m. predominó Talipariti tiliaceum var. pernambucense (Malvaceae) con árboles dispersos de Conocarpus erectus y Terminalia catappa (Combretaceae), Erythrina poeppigiana (Fabaceae) y Cecropia pittieri (Urticaceae); mientras que en el mismo gradiente altitudnal pero a unos 150 $\mathrm{m}$ de distancia de la costa se encontró una zona de crecimiento secundario donde abundaba Henriettella fascicularis (Melastomataceae), con árboles aislados de Sacoglottis holdridgei (Humiriaceae) cubiertos de epífitas en las que destacaban individuos de Guzmania sanguinea (Bromeliaceae). La densidad foliar en este gradiente fue mayor que en cualquiera de los otros pisos altitudinales.

Henriettella fascicularis (Melastomataceae) se encontró de manera abundante en el piso altitudinal intermedio, a los $200 \mathrm{~m}$ de altitud, acompañada por helechos arborescentes y terrestres, así como árboles dispersos de Clusia rosea (Clusiaceae) y Ocotea insularis (Lauraceae), al igual que palmas de Euterpe precatoria var. longivaginata. Cabe señalar que en este sitio se encontró crecimiento de Hypolytrum amplum (Cyperaceae).

En el bosque nuboso, a los $400 \mathrm{~m}$ de altitud, predominó $S$. holdridgei, acompañado de abundantes helechos arborescentes, principalmente Cyathea alfonsiana que alcanzaban alturas desde los 4 hasta los $6 \mathrm{~m}$ incrementando así la densidad foliar del sitio. Además, el sustrato estaba cubierto de melastomatáceas y helechos terrestres. En este gradiente altitudinal se encontró un bosque maduro con árboles que alcanzaron alturas de hasta $27 \mathrm{~m}$ y $114 \mathrm{~cm}$ de DAP.

No se registraron diferencias en las temperaturas por la mañana ni por la tarde entre los 200 y 400 m.s.n.m. (Tabla 1), por lo que se cree la temperatura que no es la responsable de preferencia de uso de hábitat por parte de C. ferrugineus en estos sitios, mientras que la zona altitudinal de 0 m.s.n.m. presentó una diferencia de $1.5{ }^{\circ} \mathrm{C}$ por la mañana y de $5{ }^{\circ} \mathrm{C}$ por la tarde con respecto a los otros sitios altitudinales (Tabla 1). 
Descripción del uso del hábitat: la mayor cantidad de los registros de presencia-ausencia de $C$. ferrugineus fueron en la zona altitudinal baja con un $48.8 \%$, seguido de la zona altitudinal media con $43.2 \%$ y por último la zona altitudinal alta con $8 \%$ del total de los registros. La actividad de C. ferrugineus fue mayor durante la mañana en el gradiente altitudinal intermedio y alto, con un $76 \%$ y un $100 \%$ respectivamente, mientras que a los 0 m.s.n.m. el $54.1 \%$ de los registros ocurrieron en la tarde.
No se encontraron diferencias estadísticamente significativas (Fisher, $\mathrm{P}>0.05$ ) entre las categorías de comportamiento establecidas y la hora del día, ni tampoco entre el estrato vertical utilizado por hora del día. Para las categorías de comportamiento y el estrato vertical sí se mostró diferencias significativas (Fisher, $\mathrm{P}<0.05$ ).

Todas las categorías de comportamiento en cualquiera de los estratos verticales se registraron en mayor medida por la mañana (Fig. 1A), con un $76.8 \%$ del presupuesto de
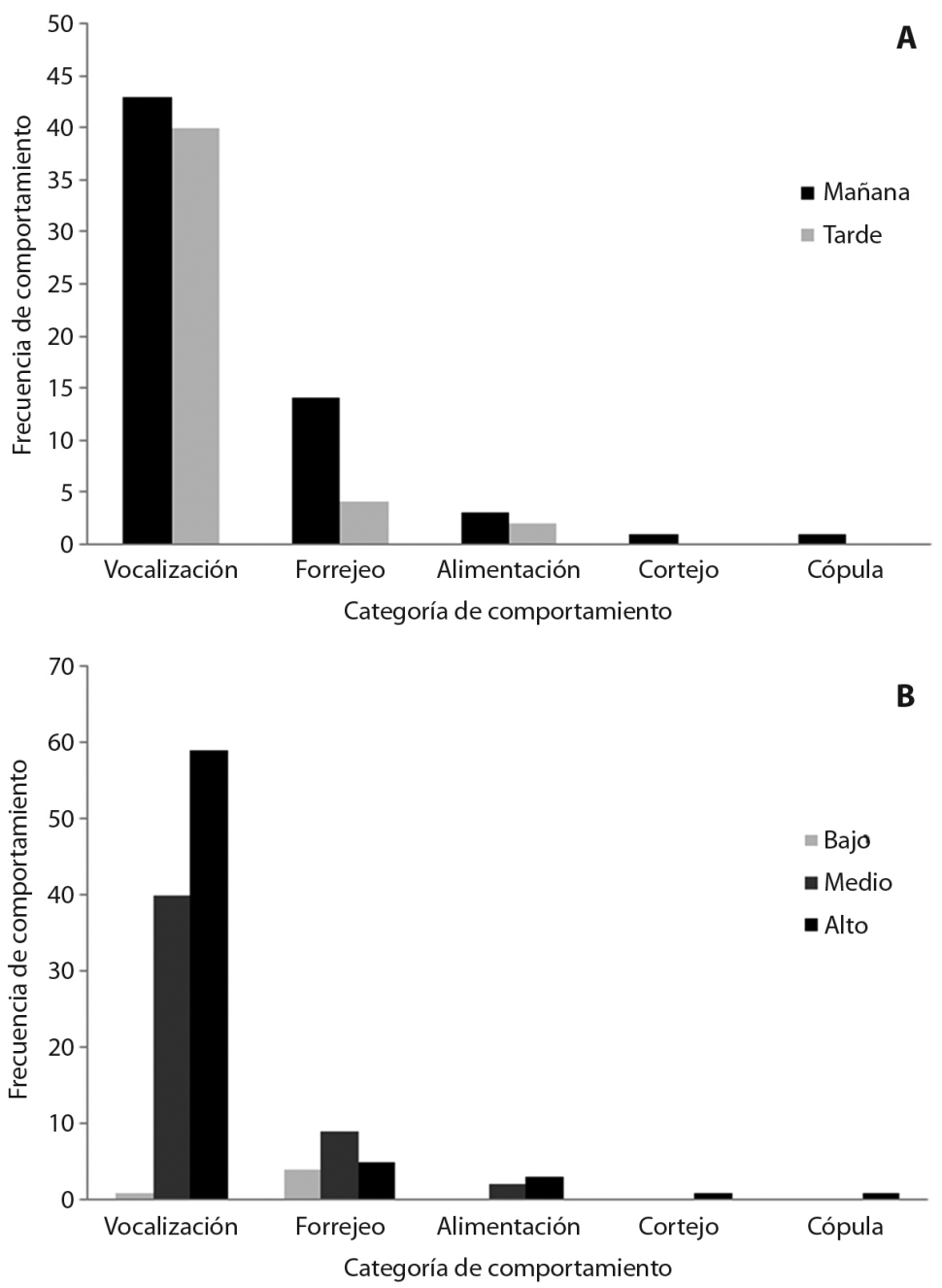

Fig. 1. Comportamiento de Coccyzus ferrugineus: A) Frecuencias de categorías de comportamiento en dependencia de la hora del día, B) Frecuencias de categorías de comportamiento en dependencia del uso de estrato vertical de la vegetación. Fig. 1. Behavior of Coccyzus ferrugineus: A) Behavior categories frequencies depending on the time of day, B) Behavior categories frequencies depending on the use of the vertical stratum of the vegetation. 
tiempo dedicado a la vocalización (Fig. 2), que fue utilizada antes o después de cualquier otro comportamiento: antes de forrajear, después de alimentarse, durante el cortejo y posterior a la cópula. El estrato alto fue el más utilizado por la especie (Fig. 3). El sotobosque resultó ser una zona de forrajeo en áreas abiertas y de cortejo en la zona donde abundaba T. tiliaceum var. pernambucense, igualmente el estratro vertical alto de la vegetación es importante para la reproducción de C. ferrugineus (Fig. 1B), así se demuestra que $C$. ferrugineus utiliza

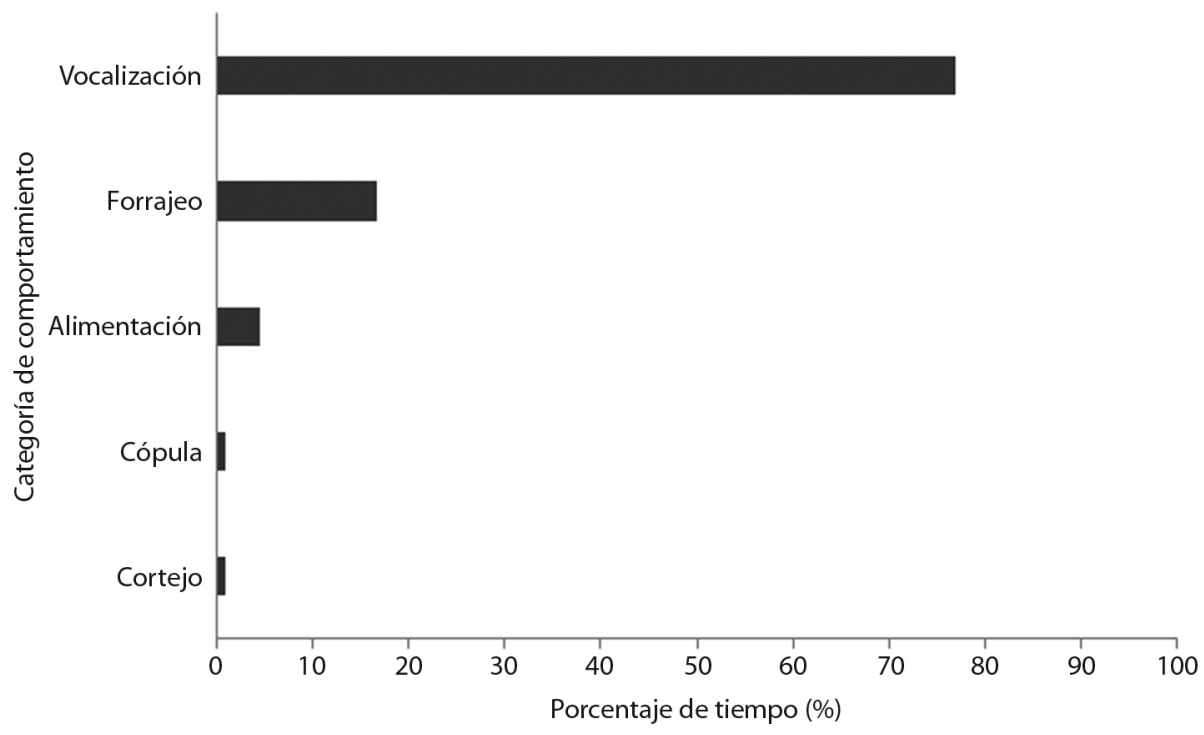

Fig. 2. Presupuesto de tiempo de Coccyzus ferrugineus durante la época reproductiva.

Fig. 2. Time budget of Coccyzus ferrugineus during the reproductive season.

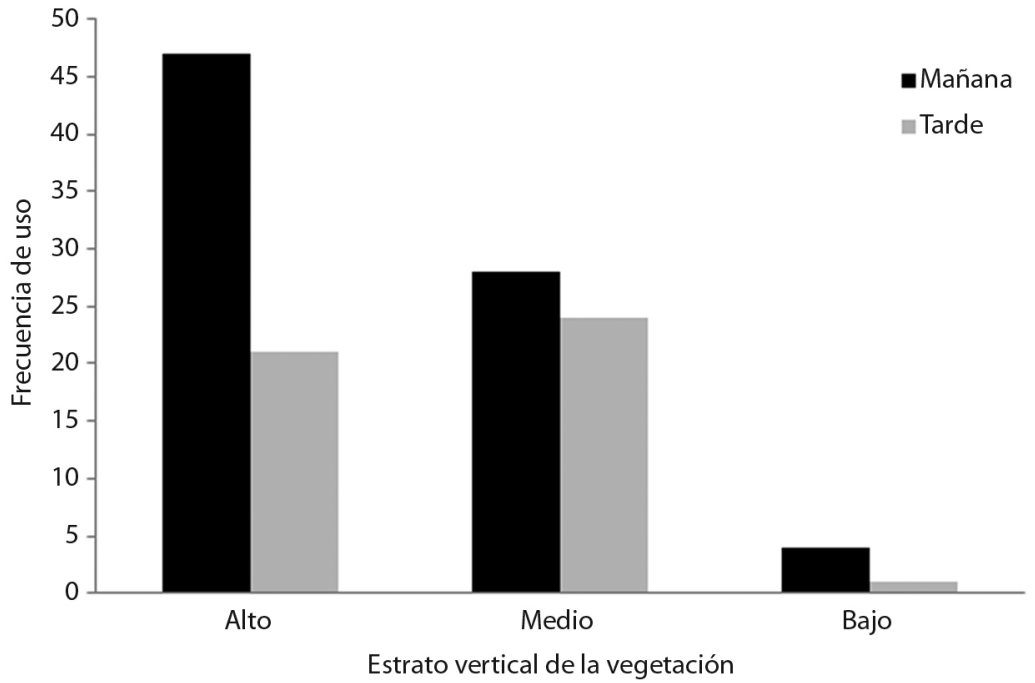

Fig. 3. Preferencia de uso del estrato vertical de la vegetación por Coccyzus ferrugineus en dependencia de la hora del día. Fig. 3. Use of the vertical stratum of vegetation preference by Coccyzus ferrugineus depending on the time of day. 
todo el estrato vertical de la estructura del bosque de la Isla del Coco, desde el sotobosque hasta el dosel.

Descripción del comportamiento de forrajeo: Durante el forrajeo, los adultos buscan intensamente alimento como invertebrados grandes, para ello utilizan el pico y realizan saltos y vuelos cortos escudriñando entre las epífitas simbiontes de $S$. holdridgei, principalmente en G. sanguinea y musgos (Fig. 4A). También buscan sus presas en foliolos o pinnas de las palmeras Cocos nucifera y Euterpe precatoria var. longevaginata, ambas de la familia Arecaceae, donde suele cazar acrídidos y blátidos (Fig. 4B). En ocasiones se detienen sobre la vegetación y exploran el sitio o descienden al suelo por presas expuestas. Pocas veces cazan insectos en el aire mediante vuelos cortos sobre el nivel del suelo, en áreas abiertas. Se observó que los adultos muestran especial preferencia por larvas de Cocytius antaeus y de Historis odius, hospederas de Annona glabra y de $C$. pittieri, respectivamente (Fig. 4C, Fig. 4D).
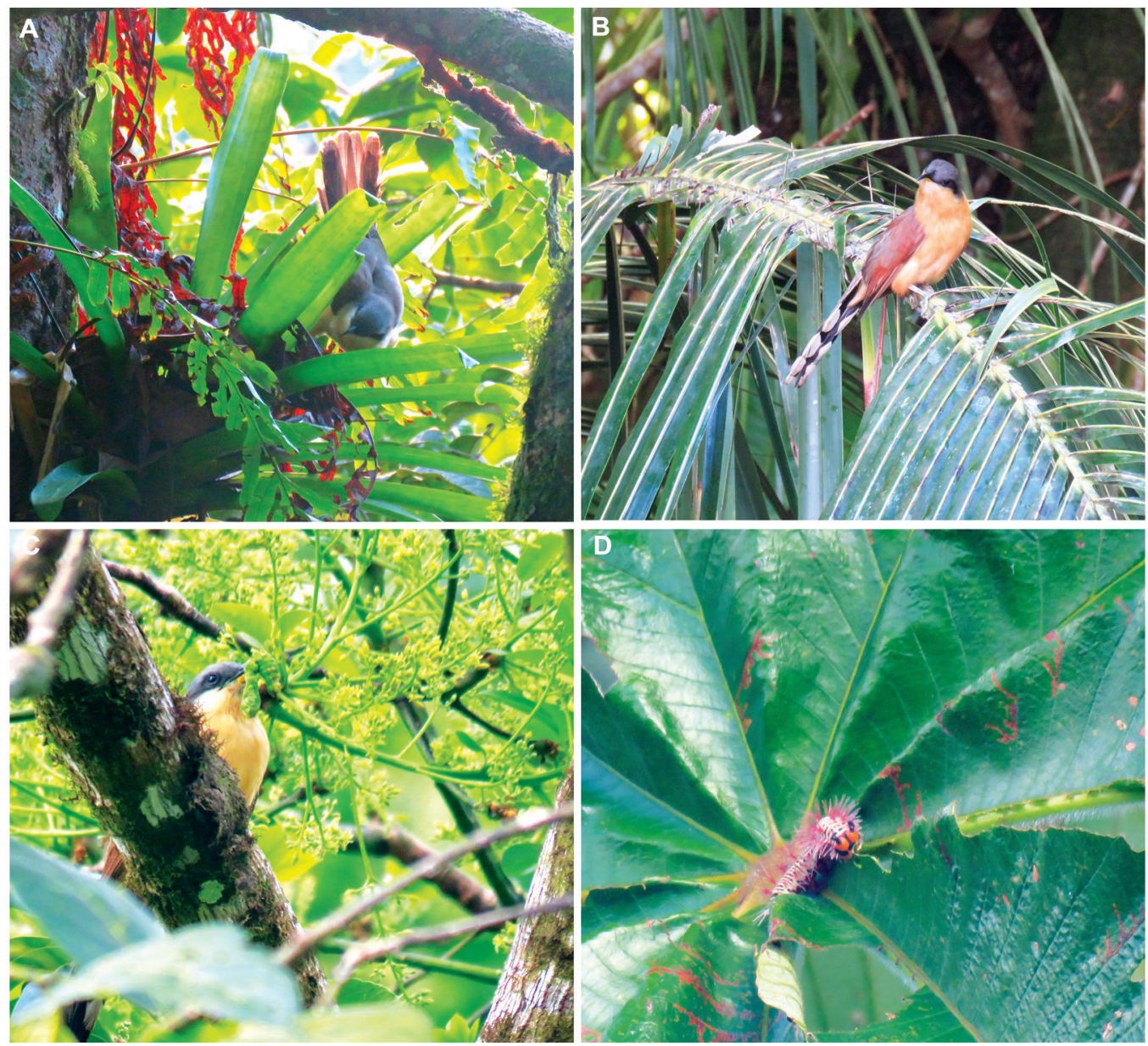

Fig. 4. A) Adulto de Coccyzus ferrugineus forrajeando en Guzmania sanguinea para alimentar al pichón. B) Adulto de C. ferrugineus forrajeando en foliolos de palmas. C) Adulto de C. ferrugineus alimentándose de larva de Cocytius antaeus. D) Larva de Historis odius en hoja de C. pittieri. Fotografías: Javier Tenorio.

Fig. 4. A) Adult of Coccyzus ferrugineus foraging in Guzmania sanguinea in order to feed the chick. B) Adult of $C$. ferrugineus foraging in palms leaflets. C) Adult of C. ferrugineus feeding on a Cocytius antaeus larva. D) Larva of Historis odius on C. pittieri leaf. Photographs: Javier Tenorio. 
TABLA 2

Fechas de registro y comportamientos de parejas reproductivas de Coccyzus ferrugineus

en el Parque Nacional Isla del Coco durante febrero y marzo de 2017

TABLE 2

Date records and behavior of breeding pairs of Coccyzus ferrugineus in Isla del Coco National Park during February and March 2017

\begin{tabular}{|c|c|c|c|c|}
\hline $\begin{array}{c}\text { Pareja } \\
\text { reproductiva }\end{array}$ & $\begin{array}{c}\text { Fecha de registro } \\
\text { (2017) }\end{array}$ & $\begin{array}{l}\text { Zona altitudinal } \\
\text { (m.s.n.m.) }\end{array}$ & Coordenadas geográficas & Comportamiento \\
\hline 1 & 14 febrero & 0 & 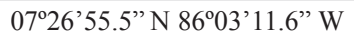 & Cortejo-cópula \\
\hline 2 & 22 febrero & 0 & $05^{\circ} 32^{\prime} 38.6^{\prime \prime} \mathrm{N} 87^{\circ} 03^{\prime} 23.6^{\prime \prime} \mathrm{W}$ & Cortejo \\
\hline 3 & 23 febrero al 3 marzo & 400 & $05^{\circ} 31^{\prime} 50.2^{\prime \prime} \mathrm{N} 87^{\circ} 04^{\prime} 010^{\prime \prime} \mathrm{W}$ & Vocalizaciones \\
\hline 4 & 25 febrero & 200 & $05^{\circ} 32^{\prime} 51.2^{\prime \prime} \mathrm{N} 87^{\circ} 02^{\prime} 51.1^{\prime \prime} \mathrm{W}$ & Cópula \\
\hline 5 & 22 febrero al 11 marzo & 0 & $05^{\circ} 32^{\prime} 39.0^{\prime \prime} \mathrm{N} 87^{\circ} 03^{\prime} 16.7^{\prime \prime} \mathrm{W}$ & Cuido parental (2 volantones) \\
\hline 6 & 7 marzo & 200 & $05^{\circ} 32^{\prime} 01.3^{\prime \prime} \mathrm{N} 87^{\circ} 03^{\prime} 03.5^{\prime \prime} \mathrm{W}$ & Cuido parental (1 volantón) \\
\hline
\end{tabular}

Mayoritariamente, las veces que se le vio forrajear en el estrato bajo fue para cazar orugas de $C$. antaeus, que se encontraban en hojas de $A$. glabra, esto porque en Bahía Wafer hay una pequeña concentración de esta especie que no supera los tres metros de altura.

Descripción del ciclo reproductivo: En total, se registraron seis parejas reproductivas, una realizando cortejo a los $0 \mathrm{~m}$ de altitud, dos parejas en cortejo-cópula (una de ellas en la zona costera y la otra a los $200 \mathrm{~m}$ altitudinales), dos con volantones (una a los $0 \mathrm{~m}$ y otra a los $200 \mathrm{~m}$ altitudinales), y una sexta pareja a los $400 \mathrm{~m}$, que se presume se encontraba en uno o más de estas categorías de comportamiento, ya que a pesar de que nunca se le logró observar directamente, sí se escuchó emitiendo vocalizaciones de contacto y cortejo (Tabla 2). Los resultados obtenidos establecen que C. ferrugineus utiliza todo el gradiente altitudinal desde el nivel del mar hasta los $400 \mathrm{~m}$ de altitud de la Isla del Coco para reproducirse.

Comportamiento de cortejo: El macho da inicio al cortejo con la búsqueda intensiva de un insecto para entregar a la hembra como regalo nupcial. La hembra lo persigue dando saltos tras él, mientras ambos emiten constantes vocalizaciones de cortejo, cortos gritos roncos y ruidosos. Una vez obtenida la presa se la muestra a la hembra y, si esta la recibe, hay cópula. Antes de la cópula el macho realiza un despliegue extendiendo su cola en abanico, exponiéndola mientras agacha su cuerpo y levanta la cola; al mismo tiempo, mantiene sus alas abiertas mostrando el rufo intenso de sus plumas alares (Fig. 5A). Cuando la hembra no se encuentra receptiva desprecia el regalo nupcial y no se da la cópula.

La pareja que se observó realizando únicamente el cortejo no copuló, ya que el macho primero cazó un grillo que al instante desechó, posteriormente cazó un segundo grillo, el cual entregó a la hembra. Sin embargo, la hembra lo dejó caer y bajó a nivel del suelo a tomarlo de nuevo. Estando ambos sobre el suelo, el macho realizó el despliegue sin éxito y la hembra se retiró del sitio con el grillo en el pico, perseguida por el macho. Este suceso se dio el 22 de febrero de 2017 en Bahía Wafer, donde abundaba $T$. tiliaceum var. pernambucense.

Las dos cópulas que se observaron se dieron en un área abierta, ambas en un árbol de $C$. pittieri, uno de $7 \mathrm{~m}$ y otro de $15 \mathrm{~m}$ de altura, los días 14 de febrero y 25 de febrero de 2017, respectivamente (Tabla 2). Durante la primera de ellas, el macho cazó un grillo en el estrato vertical bajo y se lo entregó a la hembra mientras copulaban; posteriormente se fueron saltando uno tras del otro. En la segunda, sólo la hembra vocalizaba mientras el macho buscaba 

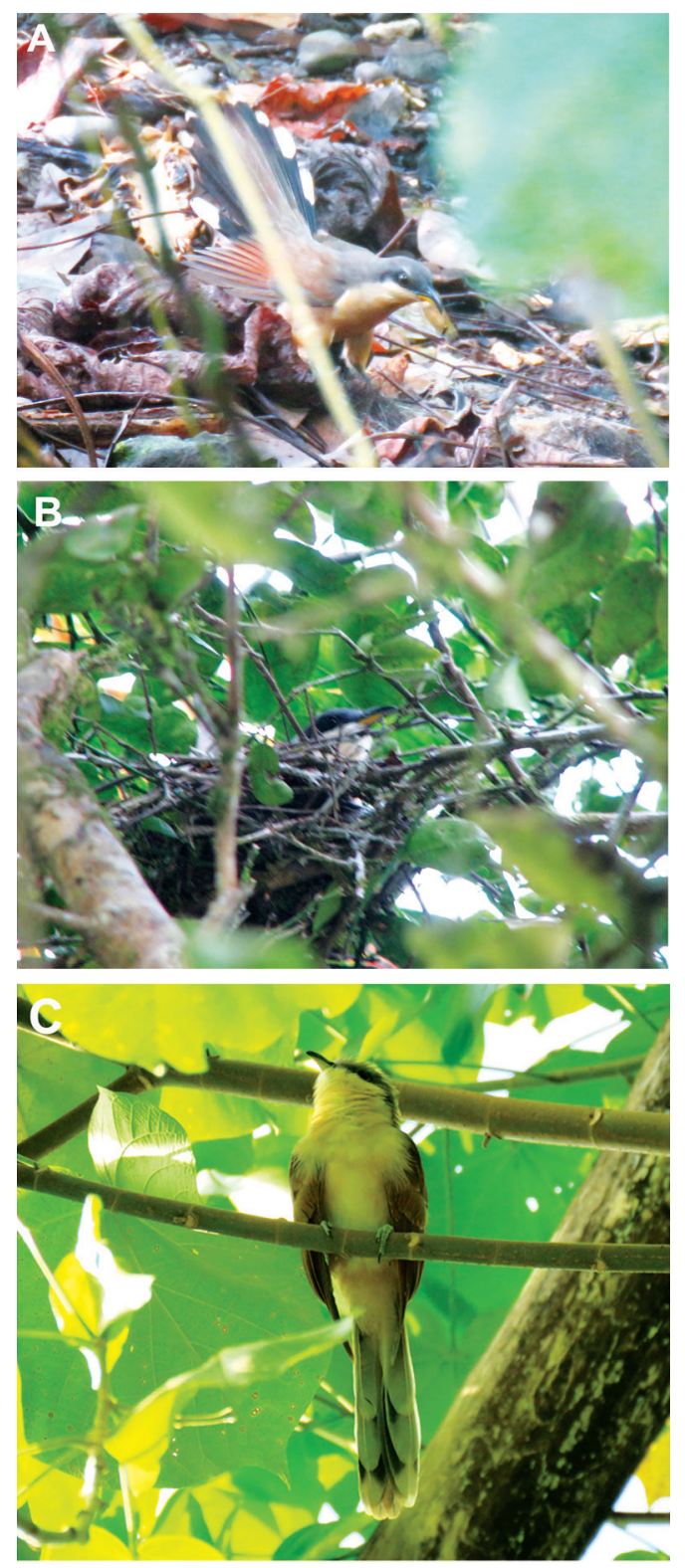

Fig. 5. A) Macho de Coccyzus ferrugineus cortejando a hembra. B) Adulto de C. ferrugineus posado sobre el nido. C) Juvenil de C. ferrugineus. Fotografías: Javier Tenorio (A, C) y Guillermo Blanco (B)

Fig. 5. A) Male of Coccyzus ferrugineus courting a female. B) Adult C. ferrugineus perched on the nest. C) Juvenile of C. ferrugineus. Photographs: Javier Tenorio (A, C) and Guillermo Blanco (B).

el regalo nupcial, este encontró una oruga y se la entregó, antes de esto el macho se alimentó de otra oruga.
Cuidado parental: Durante el período reproductivo se observó que uno de los adultos responde a los constantes llamados del volantón, que espera perchado en una rama expuesta de un árbol a una altura mayor a los $10 \mathrm{~m}$ del suelo. Esta vocalización se compone de una sola sílaba o nota de contacto breve similar a "chur$\underline{r} \underline{ }$ " que repite regularmente cada 2-5 segundos e incrementada cuando el adulto aparece hasta que es alimentado. Se observó que un solo adulto alimenta al volantón, forrajeando intensamente a vista de este, mientras éste los sigue saltando. El registro de los comportamientos de alimentación sugiere que se comunican corporalmente con movimientos de las timoneras, ya que este comportamiento se observó siempre que el adulto alimentaba al volantón.

Una vez que el adulto consigue una presa se la lleva en el pico hasta donde se encuentra el volantón y antes de entregársela la golpea y sacude repetidas veces contra una rama; mientras, el volantón incrementa sus llamados, al mismo tiempo que extiende su cola en forma de abanico y la eleva levemente. Los padres alimentan al volantón o volantones al menos dos veces por día con una sola presa por ocasión. Cuando un adulto debía hacerse cargo de dos crías repetía el mismo comportamiento con ambos para finalmente alimentarse así mismo, posteriormente abandonaba el sitio y los volantones quedaban en las perchas expuestas.

Descripción del nido: El nido de $C$. ferrugineus es en forma de taza, muy liviano. Es construido con ramitas y palitos, por lo que es endeble. El nido encontrado en enero 2011 estaba colocado sobre una ramificación secundaria de un árbol de Citrus aurantifolia (Rutaceae), a unos $2.5 \mathrm{~m}$ sobre el suelo, situado en un área abierta dentro de una matriz conformada por bosque ribereño donde el adulto tiene un ángulo de visión amplio desde el nido para la protección y vigilancia. El nido se encontraba protegido del sol por las hojas del mismo árbol. Un solo adulto se posaba sobre el nido para incubar (Fig. 5B), dejando cola y cabeza fuera del nido. Ambos sexos participan en el comportamiento de incubación. La época 
reproductiva de $C$. ferrugineus coincide con la época seca de la Isla del Coco y abarca de enero a mediados de abril. No se observó a $C$. ferrugineus parasitar nidos de otras aves.

Descripción de juveniles: Morfológicamente los inmaduros de C. ferrugineus (Fig. 5C) se diferencian de los adultos por poseer anillo ocular pálido, cabeza grisácea, que se extiende por la espalda con tintes cafés; la garganta, pecho y vientre son de color crema; con coberteras alares grisáceas pálidas y con antifaz indistinto, la mandíbula inferior amarilla pálida con frente y coronilla gris pálida. Los primeros días tienen las timoneras muy cortas y completamente blancas, a la segunda semana la cola es más larga, pero no tanto como la de un individuo adulto; además, presentan bases contrastantes indistintas o borrosas. Los adultos durante la época reproductiva presentan la mandíbula inferior y el anillo ocular amarillo anaranjado con coberteras alares rufas, vientre, garganta y pecho rojizo y las timoneras laterales negras con puntas contrastantes.

\section{DISCUSIÓN}

Durante época reproductiva la mayoría de las especies de aves disminuye su ámbito de acción por razones de cuidado parental (Stiles \& Skutch, 1989; Lloyd, 2016; Powell, Wolfe, Johnson, \& Stouffer, 2016) e incrementa la actividad vocal (Claessens, Giraud-Audine, Royer, \& Sénécaux, 2017). Considerando lo anterior es confiable decir que los sitios donde se registraron las parejas reproductivas y los inmaduros, corresponden a los sitios de anidación de C. ferrugineus (Burke, Thompson, \& Faaborg, 2017). Evidenciando que C. ferrugineus anida tanto en zonas costeras como en el bosque nuboso, al igual que en la zona intermedia de transición entre estos dos ecosistemas, desde los 0 m.s.n.m. hasta los $400 \mathrm{~m}$ de altitud.

Con respecto al ciclo reproductivo y al comportamiento de forrajeo, C. ferrugineus presentó características muy similares a otras especies del género Coccyzus (Payne \& Sorensen, 2005). Estas hacen uso de todo el estrato vertical de la vegetación, desde el sotobosque hasta el dosel, y la amplitud de uso de hábitat utilizado por ellas es variable dependiendo principalmente de la disponibilidad de presas (Toochin \& Cecile, 2014). Además, pueden hacer uso de áreas al borde de un bosque primario con dosel alto o bien utilizan bosques secundarios de dosel bajo (Claessens et al., 2017; Johnson, Hatten, Holmes, \& Shafroth, 2017).

Las variaciones anuales de temperatura en la Isla del Coco no sobrepasan $\operatorname{los} 2{ }^{\circ} \mathrm{C}$ mientras que el régimen de precipitación cambia sustancialmente, siendo los meses de enero, febrero y marzo los que presentan menor precipitación, con la mínima en febrero (Alfaro \& Hidalgo, 2016). Por el contrario durante los meses de abril a diciembre ocurren fuertes lluvias principalmente por las tardes, esto condiciona el período de anidación de C. ferrugineus y sugiere que el uso de hábitat responde a condiciones espaciotemporales y ecosistémicas, por esto la temperatura, al ser tratada como un factor aislado, no mostró ser un factor determinante para el uso de hábitat. Así, se confirma que el inicio del ciclo reproductivo es determinado por el comienzo de la época de menor precipitación en la Isla del Coco.

Una característica sobresaliente del género Coccyzus es la alimentación durante el cortejo, donde el macho demuestra sus habilidades para alimentar a los pichones lo cual constituye un carácter de selección sexual (Langridge, 1990; McNair, 1991; Claessens et al., 2017). Este evento, así como la cópula, los realizan en sitios cercanos al nido, con una ruta establecida (Toochin \& Cecile, 2015; Claessens et al., 2017; Johnson et al., 2017).

Por su parte, la construcción del nido, el árbol y el área de anidación, presentaron características similares a otras especies del género Coccyzus: distancia del nido al suelo, construcción del nido en la copa de los árboles y elección de áreas con buena visibilidad para defensa del nido (Claessens et al., 2017; Johnson et al., 2017; Lloyd, 2017). Son necesarios sitios con densidad foliar nativa alta (Toochin \& Cecile, 2015; Bond \& Glass, 2016). Por 
el contrario, muchas enredaderas, arbustos y árboles alóctonos al igual que la fragmentación de hábitat poseen efectos negativos en la distribución y anidación de la especie.

Los juveniles de C. ferrugineus son similares a los de otras especies del mismo género, manteniendo su plumaje inmaduro de dos a tres meses principalmente con diferencias en la cola y color de pico (Nolan, 1975; Toochin \& Cecile, 2015). A pesar de que se observó una diferencia de coloración en el anillo ocular y pico entre juveniles y adultos, Frieze y Lloyd (2017) indican que esto no depende de la edad del individuo sino más bien a la época reproductiva, funcionando durante la comunicación social. En las especies del género Coccyzus, la coloración del plumaje en la especie puede deberse al estadio que se encuentre el individuo, siendo determinada por el clima y gradiente ambiental (Lloyd, 2016).

Según Sealy (2003), y Johnson et al. (2017), la disponibilidad de presas para especies del género Coccyzus tiene una relación inversa con respecto a la distancia de cuerpos de agua y humedad; es por ello que se deben estudiar las condiciones microclimáticas para determinar las características y dinámica del sitio en función de C. ferrugineus (DuránQuesada \& Alfaro, 2015). Coccyzus ferrugineus utiliza sitios costeros como áreas de forrajeo y anidación, probablemente porque tienen una elevada disponibilidad de presas a un costo energético bajo en comparación con áreas de mayor altitud, donde la vegetación es más densa y la búsqueda posiblemente más desgastante. Por estas mismas razones el ciclo reproductivo de $C$. americanus requiere hábitats húmedos (Russell \& Holmes, 2015; Friggens \& Finch, 2015).

La estructura y riqueza de la vegetación encontrada coincide con lo descrito por Montoya (2016), caracterizada por el Bosque Tropical Lluvioso (Tropical Rain Forest) y Bosque Tropical Nuboso (Tropical Cloud Forest), y una marcada diferenciación ecosistémica (Fourriére et al., 2016). En zonas altitudinales intermedias y altas, $C$. ferrugineus forrajeará principalmente en epífitas ya que son muy abundantes en los árboles autóctonos, mientras que en la zona altitudinal baja la heterogeneidad estructural de plantaciones, áreas abiertas y zona costera le permite a $C$. ferrugineus utilizar los tres estratos verticales (Powell et al., 2016). Además, en la zona baja $A$. glabra facilita el forrajeo de $C$. ferrugineus en el estrato vertical bajo, ya que $C$. antaeus es hospedera de este árbol (Montoya, 2009). Posiblemente, las diferencias observadas en el uso que $C$. ferrugineus le da a cada estrato vertical correspondan a la disponibilidad de presas y a la adaptación individual a la zona habitada.

De esta manera, es importante conocer los patrones de desplazamientos de C. ferrugineus, ya que la acumulación de sus movimientos conduce a la dinámica de poblaciones y determina el flujo genético (Farrel, 2013; Powell et al., 2016; Johnson et al., 2017). Además, al tratarse de un ecosistema fragmentado por introducción de especies exóticas, el incremento de áreas con alta densidad de arbustos y vegetación alóctona podrían provocar el decline poblacional de C. ferrugineus.

La distribución y abundancia de C. ferrugineus en zonas altitudinales bajas podría obedecer a la densidad superior de depredadores en zonas intermedias y altas (Orias, 2012). Es bien conocida la amenaza de las especies alóctonas sobre aves insulares en el mundo (Jones et al., 2016; Zarzoso-Lacoste et al., 2016), en el caso de la Isla del Coco se ha demostrado que ratas (Rattus rattus y R.novergicus) y gatos ferales (Felis catus) depredan a C. ferrugineus y se aprovechan de la época de anidación de las aves (Orias, 2012). Por ello, es urgente evaluar impactos en la dinámica poblacional de la presa (Bianco, Soto, Vargas, \& PerézEnciso, 2015; Zarzoso-Lacoste et al., 2016; Woinarski et al., 2017).

Sin embargo, la falta de conocimiento imposibilita establecer relaciones ecológicas con las especies exóticas que habitan la Isla del Coco. A pesar de que se conoce que depredan aves, tanto adultas como juveniles y huevos, se desconoce la densidad poblacional que está siendo consumida por estos depredadores (Orias, 2012), así como su tasa de 
reclutamiento. Además, parte importante de la dieta de las ratas incluye a las mismas presas de $C$. ferrugineus (inclusive insectos en estado larval del orden Lepidoptera), e impiden el desarrollo de árboles endémicos, importantes para el ciclo reproductivo de C. ferrugineus, por ingesta de frutos como el de S. holdridgei (Orias, 2012).

Otras especies del género Coccyzus actualmente se encuentran amenazadas por invasión de especies alóctonas tanto vegetales como animales, así como por el cambio climático, que afecta negativamente a las presas de estas especies (Friggens \& Finch, 2015). Por ello, estudios sobre la ecología de $C$. ferrugineus en base a los requerimientos de hábitat de la especie, así como de su densidad poblacional, son indispensables para categorizar las amenazas que le afectan y para poder ejercer un plan de manejo que incluya áreas de reproducción y programas de restauración ecológica (Woinarski et al., 2017).

Actualmente, el principal obstáculo para ejercer planes de manejo que beneficien a $C$. ferrugineus es la falta de conocimiento sobre su ecología. Se recomienda realizar estudios espaciotemporales sobre el ámbito de acción, modelando asociaciones de hábitat en combinación con anillamiento y/o acústica y así conocer la tolerancia de la especie ante la destrucción del hábitat que sufre actualmente (Burke et al., 2017; Toochin \& Cecile, 2015; Powell et al., 2016; Lloyd, 2017). Este estudio representa la base para el conocimiento de la biología reproductiva, requerimientos de hábitat y manejo de C. ferrugineus (BirdLife, 2019; Payne, 2019; Schulenberg, 2019).

Declaración de ética: los autores declaran que todos están de acuerdo con esta publicación y que han hecho aportes que justifican su autoría; que no hay conflicto de interés de ningún tipo; y que han cumplido con todos los requisitos y procedimientos éticos y legales pertinentes. Todas las fuentes de financiamiento se detallan plena y claramente en la sección de agradecimientos. El respectivo documento legal firmado se encuentra en los archivos de la revista.

\section{AGRADECIMIENTOS}

Agradecemos a la Asociación Ornitológica de Costa Rica por aportar fondos y apoyar esta investigación, al Área de Conservación Marina Isla del Coco y a los funcionarios del Parque Nacional Isla del Coco por el apoyo brindado, en especial a Guillermo Blanco por sus valiosos aportes y a Esteban Herrera por su apoyo en logística. De igual manera agradecemos la ayuda brindada por Fabián Monge, Diego Morales y Andrey Acosta.

\section{RESUMEN}

Introducción: Coccyzus ferrugineus es el ave endémica menos estudiada de la Isla del Coco se desconocen muchos aspectos sobre su historia natural y comportamiento, siendo de las especies menos conocidas en Costa Rica. Objetivo: Describir el comportamiento reproductivo, de forrajeo, y el uso de hábitat de C. ferrugineus para generar información para la toma de decisiones de conservación de la especie. Métodos: Se hicieron observaciones directas de comportamiento (forrajeo, cortejo, vocalización, alimentación y cópula) durante dos periodos de observación (mañana y tarde), en parcelas entre los 0-400 m de altitud, clasificando el estrato de vegetación (bajo-medio-alto) para estudiar el uso de hábitat. Se presenta una descripción del comportamiento de forrajeo y del ciclo reproductivo. Resultados: Se demuestra que $C$. ferrugineus utiliza todo el gradiente altitudinal de la Isla del Coco, desde el nivel del mar hasta los $400 \mathrm{~m}$ de altitud en el bosque nuboso, para reproducirse y su época reproductiva está marcada por el inicio de la época seca. No se encontraron diferencias significativas entre las categorías de comportamiento establecidas y la hora del día (Fisher, $\mathrm{P}>0.05$ ). Sin embargo sí se mostró diferencias significativas para las categorías de comportamiento y el estrato vertical (Fisher, $\mathrm{P}<0.05$ ) demostrando que utiliza todo el estrato vertical de la estructura vegetal. Conclusiones: Este estudio representa la base para el conocimiento de la biología reproductiva e historia natural de $C$. ferrugineus, es urgente realizar más estudios sobre esta especie para poder protegerla y conservar el ecosistema terrestre de la Isla del Coco.

Palabras clave: Comportamiento reproductivo; uso de hábitat; Isla del Coco; Coccyzus ferrugineus; endemismo; Costa Rica; conservación. 


\section{REFERENCIAS}

Acosta-Vargas, L. (2016). Population status of the tree Sacoglottis holdridgei (Humiriaceae) at Isla del Coco National Park, Costa Rica. Revista de Biología Tropical, 64(Supplement 1), S263-S275.

Alfaro, E. J., \& Hidalgo, H. G. (2016). Climate of an oceanic island in the Eastern Pacific: Isla del Coco, Costa Rica, Central America. Revista de Biología Tropical, 64(Supplement 1), S59-S74.

Altmann, J. (1974). Observational study of behavior: sampling methods. Behaviour, 49(3), 227-266.

Avalos, V. D. R. (2009). Aspectos del comportamiento de forrajeo de Phibalura flavirostris boliviana (Cotingidae, Passeriformes). Ecología en Bolivia, 44(1), 62-66.

Bellon, H., Saenz, R., \& Tournon, J. (1983) K-Ar radiometric ages of lavas from Cocos Island (Eastern Pacific). Marine Geology, 54, 17-23

Bianco, E., Soto, H. W., Vargas, L., \& Pérez $\square$ Enciso, M. (2015). The chimerical genome of Isla del Coco feral pigs (Costa Rica), an isolated population since 1793 but with remarkable levels of diversity. Molecular Ecology, 24(10), 2364-2378.

BirdLife International (2019). Species factsheet: Coccyzus ferrugineus. BirdLife International IUCN Red List for birds. Cambridge, UK. Recuperado de http:// www.birdlife.org

Bond, A. L., \& Glass, T. (2016). First record of Yellowbilled Cuckoo Coccyzus americanus on Tristan da Cunha, South Atlantic Ocean. Bulletin of the British Ornithologists' Club, 136(3), 214-216.

Burke, A. D., Thompson III, F. R., \& Faaborg, J. (2017). Variation in early-successional habitat use among independent juvenile forest breeding birds. The Wilson Journal of Ornithology, 129(2), 235-246.

Claessens, O., Giraud-Audine, M., Royer, F., y Sénécaux, L. (2017). What is the breeding range and breeding season of Pearly-breasted Cuckoo Coccyzus euleri? New records and breeding in French Guiana. Bulletin of the British Ornithologists ' Club, 137(1), 3-11.

Cueto, V. R. (1996). Relación entre los ensambles de aves y la estructura de la vegetación: un análisis a tres escalas espaciales. (Tesis de Doctorado). Universidad de Buenos Aires, Argentina.

Dawson, J., Oppel, S., Cuthbert, R. J., Holmes, N., Bird, J. P., Butchart, S. H., ... \& Tershy, B. (2015). Prioritizing islands for the eradication of invasive vertebrates in the United Kingdom overseas territories. Conservation Biology, 29(1), 143-153.

Durán-Quesada, A. M., \& Alfaro, E. (2016). A multiscale analysis of moisture supply associated with precipitation on Isla del Coco, Costa Rica. Revista de Biología Tropical, 64(Supplement 1), S87-S103.

Farrell, L. L. (2013). Examining the genetic distinctiveness of the western subspecies of Yellow-billed Cuckoo Coccyzus americanus occidentalis. Ardea, 101(2), 165-170.

Fourriére, M., Azofeifa-Solano, J. C., \& Sandoval, L. (2016). Species richness and density of seabirds in Isla del Coco bays, Costa Rica, related to the occurrence of breeding colonies. Marine Ornithology, 44, 241-246.

Frieze, R. D., \& Lloyd, J. D. (2017). Eye-ring coloration is not a reliable indicator for aging Mangrove Cuckoos (Coccyzus minor). The Wilson Journal of Ornithology, 129(1), 163-165.

Friggens, M. M., \& Finch, D. M. (2015). Implications of climate change for bird conservation in the southwestern US under three alternative futures. PloS One, 10(12), 1-22.

Hughes, J. M. (2006). Phylogeny of the cuckoo genus Coccyzus (Aves: Cuculidae): a test of monophyly. Systematics and Biodiversity, 4(4), 483-488.

Johnson, M. J., Hatten, J. R., Holmes, J. A., y Shafroth, P. B. (2017). Identifying western yellow-billed cuckoo breeding habitat with a dual modelling approach. Ecological Modelling, 347, 50-62.

Jones, H. P., Holmes, N. D., Butchart, S. H., Tershy, B. R., Kappes, P. J., Corkery, I., ... \& Campbell, K. (2016). Invasive mammal eradication on islands results in substantial conservation gains. Proceedings of the National Academy of Sciences, 113(15), 4033-4038.

Langridge, H. (1990). Courtship feeding behavior in the Mangroove Cuckoo (Coccyzus minor). Florida Field Naturalist, 18(3), 55-56.

Lehner, P.N. (1996). Handbook of Ethological Methods. Cambridge, Reino Unido: Cambridge University Press.

Lloyd, J. D. (2016). Phenotypic Variation in Mangrove Cuckoo (Coccyzus minor) across Its Geographic Range. PloS One, 11(3), 1-15.

Lloyd, J. D. (2017). Movements and use of space by Mangrove Cuckoos (Coccyzus minor) in Florida, USA. PeerJ, 5, 1-14.

Martínez, C. G. H., Montoya, M., \& Camacho, P. (2011). Comportamiento reproductivo y alimentario de $\mathrm{Coc}$ cyzus ferrugineus (Cuculidae), en el Parque Nacional Isla del Coco, Costa Rica. Brenesia, 75-76, 112-114.

Martin, P., \& Bateson, P. (2007). Measuring Behaviour: An Introductory Guide. Cambridge, Reino Unido: Cambridge University Press. 
McNair, D. B. (1991). Copulation in the mangrove cuckoo (Coccyzus minor). Florida Field Naturalist, 19, 84-85.

Medina, F. M., Bonnaud, E., Vidal, E., \& Nogales, M. (2014). Underlying impacts of invasive cats on islands: not only a question of predation. Biodiversity and Conservation, 23(2), 327-342.

Montoya, M. (2004). Vertebrados terrestres alóctonos de la Isla del Coco, Costa Rica. San José, Costa Rica: Fundación Amigos de la Isla del Coco/Área de Conservación Marina Isla del Coco.

Montoya, M. (2009). Insecta, Lepidoptea, Sphingidae, Cocytius anteus Drudy. First record for Isla

del Coco, Costa Rica and notes on its hostplant. Check List, $5(2), 151-153$.

Montoya, M. (Ed.). (2016). Costa Rican Ecosystems. Chicago, USA: The Chicago University Press.

Nolan, V. (1975). External differences between newly hatched cuckoos (Coccyzus americanus and $C$. erythropthalmus). Condor, 77(3), 341.

Orias, N. (2012). Dieta de ratas (Rattus rattus y $R$. norvegicus) introducidas y gatos (Felis catus) introducidos en el Parque Nacional Isla del Coco y su relación con las tres especies de aves endémicas. (Tesis de maestría). Universidad Nacional, Heredia, Costa Rica.

Payne, R. B., \& Sorensen, M. D. (2005). The cuckoos (Vol. 15). New York: Oxford University Press Inc.

Payne, R. (2019). Cocos Cuckoo (Coccyzus ferrugineus). In J. del Hoyo, A. Elliott, J. Sargatal, D. A. Christie, \& E. de Juana (Eeds.). Handbook of the Birds of the World Alive. Barcelona, España: Linx Editions. Recuperado de https://www.hbw.com/node/54894

Powell, L. L., Wolfe, J. D., Johnson, E. I., \& Stouffer, P. C. (2016). Forest recovery in post-pasture Amazonia: Testing a conceptual model of space use by insectivorous understory birds. Biological Conservation, 194, 22-30.

Ralph, C. P. (1975). Life style of Coccyzus pumilus, a tropical cuckoo. The Condor, 77(1), 60-72.

Russell, J. C., \& Holmes, N. D. (2015). Tropical island conservation: rat eradication for species recovery. Biological Conservation, 185, 1-7.
Schulenberg, T. S. (2019). Cocos Cuckoo (Coccyzus ferrugineus). In T. S. Schulenberg (Ed.), Neotropical Birds Online Cornell Lab of Ornithology. Ithaca, NY: Cornell Lab of Ornithology. Recuperado de Neotropical Birds Online: https://neotropical.birds.cornell.edu/ Species-Account/nb/species/coccuc1

Sealy, S. G. (2003). Laying times and a case of conspecific nest parasitism in the Black-billed Cuckoo. Journal of Field Ornithology, 74(3), 257-260.

Slud, P. (1964). The birds of Costa Rica: distribution and ecology. Las aves de Costa Rica: distribución y ecología. Bulletin of the American Museum of Natural History, 128, 1-430.

Stiles, G., \& Skutch, A. F. (1989). Guía de aves de Costa Rica. Heredia, Costa Rica: Editorial INBio.

Tebbich, S., Taborsky, M., Fessl, B., Dvorak, M., y Winkler, H. (2004). Feeding behavior of four arboreal Darwin's finches: adaptations to spatial and seasonal variability. The Condor, 106(1), 95-105.

Toochin, R., \& Cecile, D. (2014). Status and occurrence of Yellow-billed Cuckoo (Coccyzus americanus) in British Columbia. Recuperado de http://linnet. geog.ubc.ca/biodiversity/efauna/documents/Yellowbilled_Cuckoo-RT-DC-g.pdf

Trusty, J. L., Kesler, H. C., \& Delgado, G. H. (2006). Vascular flora of Isla del Coco, Costa Rica. Proceedings of the California Academy of Sciences, 57, 247.

Woinarski, J. C. Z., Murphy, B. P., Legge, S. M., Garnett, S. T., Lawes, M. J., Comer, S., ... \& Paton, D. (2017). How many birds are killed by cats in Australia? Biological Conservation, 214, 76-87.

Zamora, A. (2008). Estructura y composición florística de los bosques de bahía en el Parque Nacional Isla del Coco Área de Conservación Marina Isla del Coco. (Práctica de Especialidad). Instituto Tecnológico de Costa Rica, Cartago, Costa Rica.

Zarzoso-Lacoste, D., Bonnaud, E., Corse, E., Gilles, A., Meglecz, E., Costedoat, C., ... \& Vidal, E. (2016). Improving morphological diet studies with molecular ecology: an application for invasive mammal predation on island birds. Biological Conservation, 193, 134-142. 